\title{
THE APPLICATION OF WAP AND WEBGIS IN THE SYSTEM OF INSECTS' CLASSIFICATION RETRIEVAL
}

\author{
Qing Zou ${ }^{1}$, Lin Huang ${ }^{1, *}$, Xuexia Wang ${ }^{1}$, Dongmei Lang ${ }^{2}$ \\ ${ }^{1}$ College of Information Engineering, Northwest A \& F University, Yangling, Shaanxi, China, \\ 712100 \\ ${ }^{2}$ College of Changzhi, Changzhi, Shanxi, China, 046011 \\ * Corresponding author, Address: College of Information Engineering, Northwest A \& F \\ University, Yangling, Shaanxi, China, 22 Xinong Road, 712100, P. R. China, Tel: +86-29- \\ 87091546, Fax: +86-29-87092353,Email: hl@nwsuaf.edu.cn
}

Abstract: This paper mainly introduced how WAP, Google API and 51ditu API were applied to the System of Insects' Classification Retrieval. The introduction of Google API makes the query interface more visualized, convenient and humane. The purpose of 51ditu API is to provide support for decision-making when open country investigators are considering investigation routes. The application of WAP enables to extend the scope of the functions of the retrieval system from computer to cell phone, which renders more conveniences to open country investigators to access this system.

Keywords: Insects' Classification Retrieval, WEBGIS, WAP

\section{INTRODUCTION}

In these days, as computer technology has rapidly advanced, computer has been applied in every field of science and technology. Especially, the advent of computer network technologies has significantly changed the ways people live and work. However, the application of computer technology in the fields of identification and retrieval of insects still comparatively lags behind as many experts and workers (especially domestic) in the mentioned fields are still using the traditional ways to work and study, which will definitely have a strong negative impact on the advance of the science research. Although many researchers both here and abroad are dedicated to the application of

Zou, Q., Huang, L., Wang, X. and Lang, D., 2008, in IFIP International Federation for Information Processing, Volume 259; Computer and Computing Technologies in Agriculture, Vol. 2; Daoliang Li; (Boston: Springer), pp. 809-816. 
the technologies in this field, the introduction and use of them are far from being sufficient and meeting the needs of the societies.

In the past ten more years, entomologist in China has developed many expert systems for assistant identification of insects. For example, Lu Huimeng has developed the Design and Complementation of Expert System for Classification Retrieval and Diagnosis for China ACRIDOIDEA (ESCA) (Lu et al., 2003) and Lucid: A Multi-way Expert System for Identification and Diagnosis (Sun et al., 2002). Both are only for stand-alones and not available for different persons simultaneously. There is also some web-based query systems for classification such as Design and Development of Web System for Isoptera Taxonomy (Xu et al., 2004) developed by the Research Center of City Insects of Zhejiang University. Nevertheless, such a system includes little new technology, has an insufficient function, covers a small scope of the related area, and so is unable to eliminate the inconveniences occurring in fieldwork when using a retrieval system. To solve this problem, this thesis pictures how WAP and WEBGIS are applied and actualized in retrieval system for entomologic classification. The introduction of WEBGIS makes the query interface more visualized, convenient and humane and provide support for decision-making when open country investigators are considering investigation routes. The application of WAP enables to extend the scope of the functions of the retrieval system from computer to cell phone, which renders more conveniences to open country investigators to access this system.

\section{THE APPLICATION OF WEBGIS}

\subsection{Google API}

The System of Insects' Classification Retrieval Based on WEBGIS provided users three ways to query: basic query, advanced and regional query. Google API is basically meant for regional query which including two functions: providing the queried information of insect distribution in a certain region and of distribution of a certain insect.

Google maps API can display in map information of a designated region by loading XML files, which are used to describe the regional information. By constructing the XML files describing the regional insect distribution and through Google maps API, the distribution region of insects can be visualized in the map. 


\subsubsection{Query of the Information of a Regional Insect Distribution}

Actualization is demonstrated by Fig. 1 as follow.

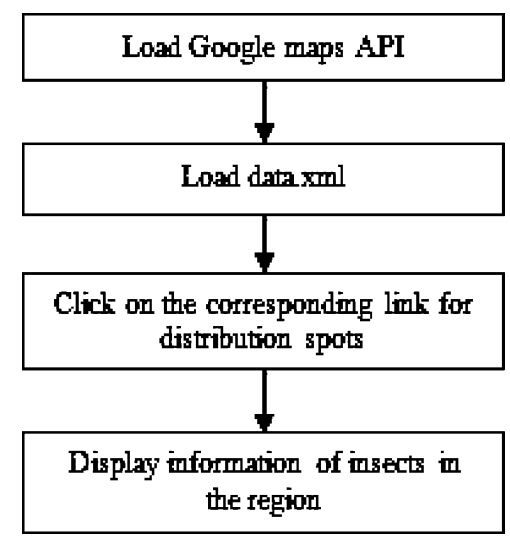

Fig. 1. Query of information of regional insect distribution

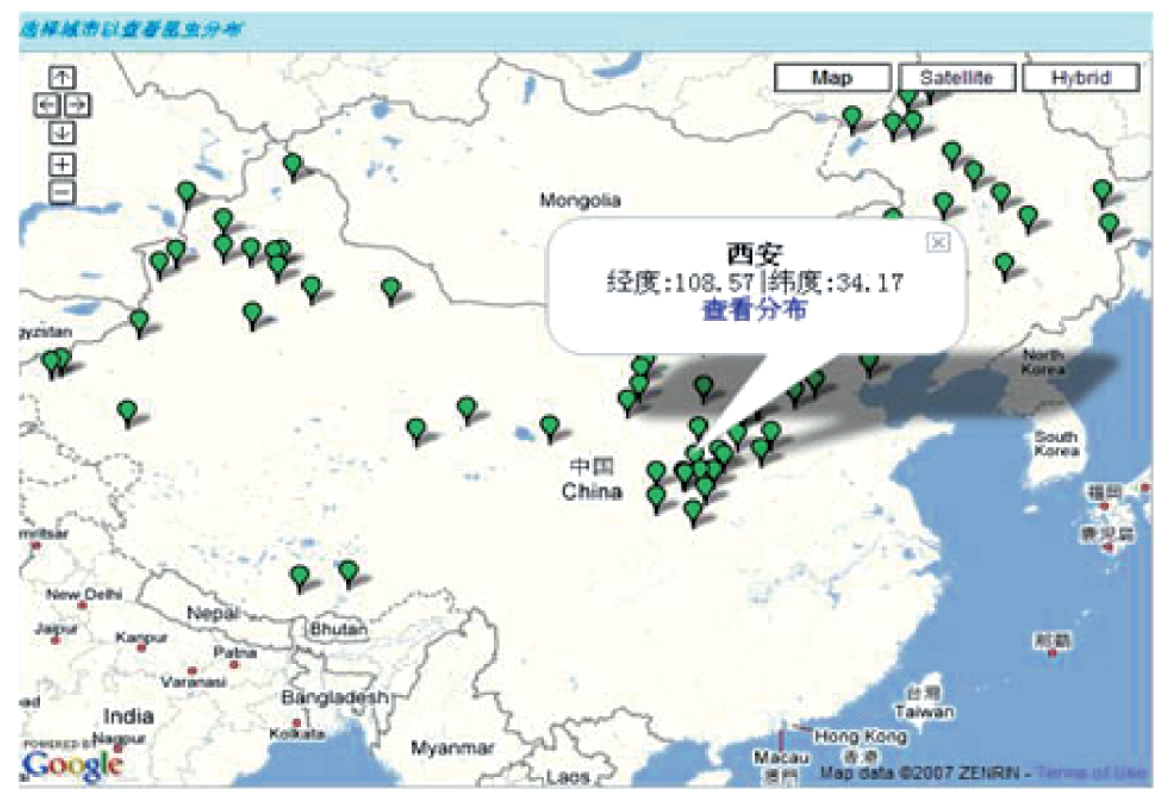

Fig. 2. Display of the region of insect distribution

In the process of query, the region of insect distribution is comparatively stable, so its information should be saved in a fixed XML file (data.xml), or such a file will be created termly from the datasheets containing insect information. System will display the insect distribution spots in the map by 
data.xml. When the user's mouse is pointed at a certain distribution spot, the system will display the information of the geographical location of that spot, and provide a query link "consult distribution" (Fig. 2); when the user clicks on the link, the system will extract the information of the distribution spot, and then retrieve information of all the insects in the region from the database and display it to the user.

\subsubsection{Query of the Insect Distribution of the Designated Species}

When using this query, the related information (the scientific names in Chinese or Latin or local) of the species is entered by the user, then the distribution of a designated insect is retrieved from the database, and a tmp.xml including information of the distribution region of the insect is created in a dynamic way, and finally, by loading Google maps API, the distribution spot will be displayed in the map. The actualization is demonstrated in Fig. 3 as follow.

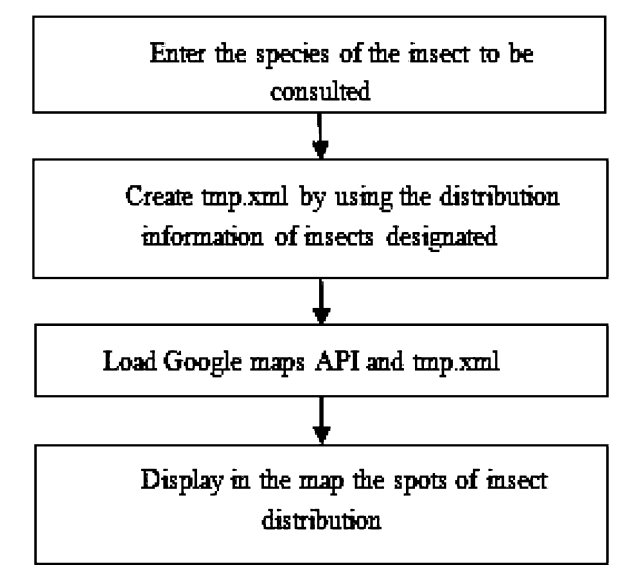

Fig. 3. Query of the distribution region of designated insects

\section{$2.2 \quad$ 51ditu API}

Research of insect distribution requires frequent field trip to other places in the country. The system has the function of displaying the dynamic trip routes in the map at the user's convenience when the user enters a start point and end point (including midway station).

This function developed through 51ditu API, an interface of WEBGIS. When the user picks a city as the destination of investigation listed in the table of option of cities, red round dots will flash signaling as chosen cities. 
When clicking on "displaying the routes", there will be lines linking all the chosen cities. When clicking on any line between any two cities, the total distance (specifically, the length of the railways between cities) of the field trip will be displayed. Besides, for the convenience, the system offers future 72 hour weather forecast for the national major cities. The result is demonstrated in Fig. 4 as follow.

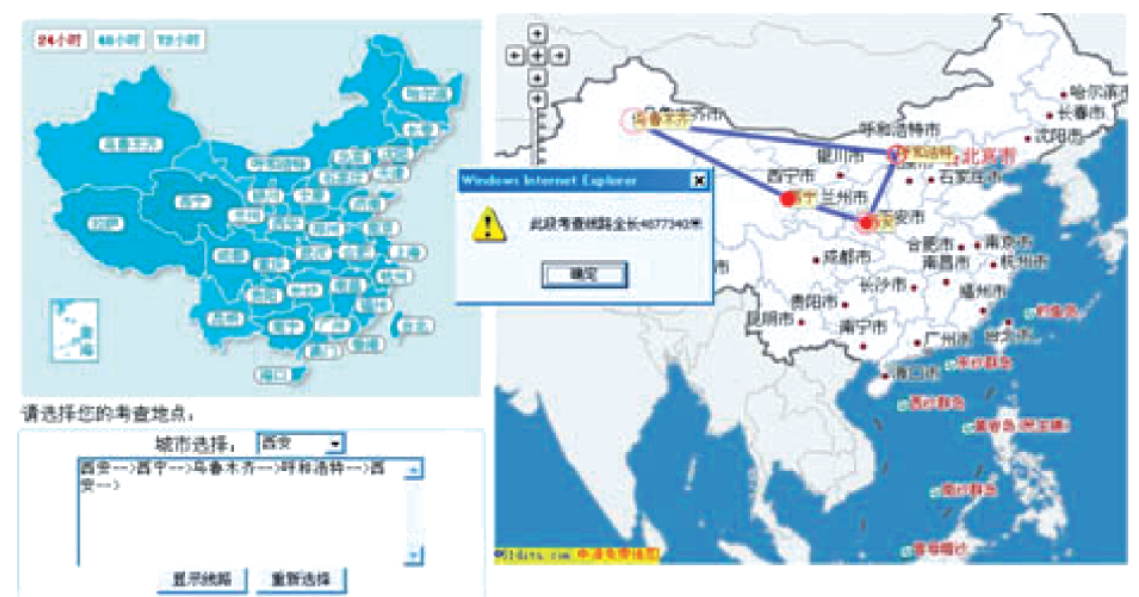

Fig. 4. Field trip route

\subsection{The application of WAP}

The popularization of WAP phone makes mobile phones possible to share computer network information. In regard with the mass tool-mobile phone, this system adopts WEB and WAP as means for users to share information of insect data on servers, so that users can not only communicate with servers through WWW, but log on WAP with a mobile phone at convenience of those who have no access to WWW. Therefore, constraint of time and space dissolves and complete sharing of information is achieved.

In the Webpage for a mobile phone to log on WAP, the primary functions of query and expert diagnosis on insect information are achieved.

\subsubsection{Retrieval on WAP Phone}

The mobile phone provides fuzzy query. When using this function, the user may enter a complete insect name in Chinese or Latin, or may enter any characters of a complete name. The homepage of WAP and the results of query are shown as following Fig. 5 and Fig. 6. 


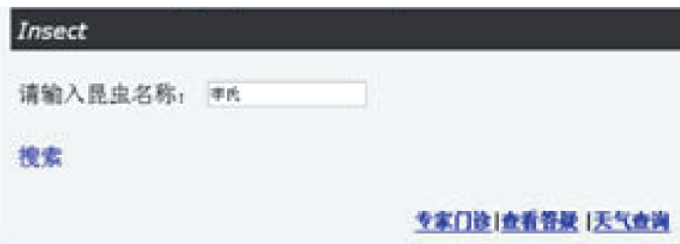

Fig. 5: WAP Homepage

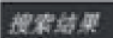

㙯大是䭪

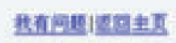

Fig. 6. Results of WAP query

\subsubsection{Expert Diagnosis}

When using this system, if there could be any professional problem, the user may ask questions by using BBS, which could be solved by experts online. And feedbacks could be presented through posts any time when users feel the system functions are insufficient and need to be improved so that the system management committee could be able to consider advice or ideas in time and get the system improved.

The interface of cell phone expert diagnosis is shown in Fig. 7 as following.

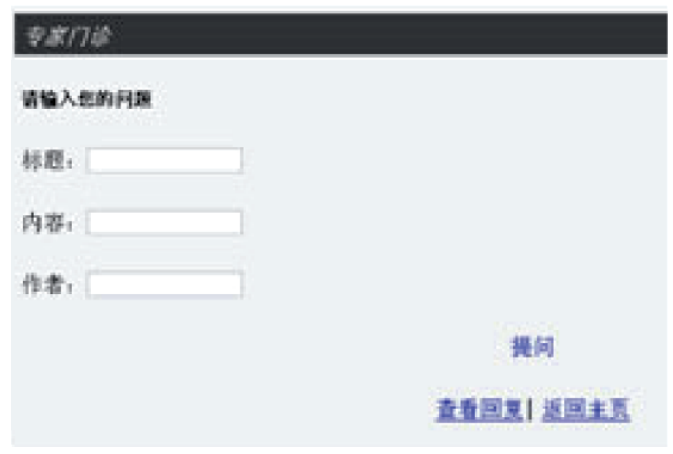

Fig. 7. Expert diagnosis of WAP

\section{DISCUSSIONS}

This thesis introduces the application of WEBGIS and WAP in the System of Insects' Classification Retrieval and the approaches of actualization of 
them. However, there are still a couple of problems found in the process of the actualization of the system.

The First problem is the display level of the insect distribution region in the map. Because the data of domestic geographic information and information for insect distribution in hand are not exclusive enough, the displayed spots can only represent regions at a municipal level instead of smaller ones.

The second is a trip route made. When the start point and end point are entered, the system can display in the map the dynamic travel routes. However, the spots entered are confined in cities at least at municipal level. Further study is required when more detailed spots are dealt. Furthermore, since at present 51ditu API interface can only provide addition of polylines connecting two places and displaying the distance of the road, which fails to picture the particular routes for transport vehicles in visualized way in the map.

The last is images displayed on mobile phone. Viewing a picture is related to the internet speed (the actual speed is between 20 to $40 \mathrm{~K}$ ), then cell phone signals and situation of network. The test results show that, to display a bigger, more memory demanding map, a higher WAP speed is required. The existing one is a major problem.

\section{CONCLUSION}

The application of WEBGIS and WAP in the System of Insects' Classification Retrieval provides the user a new retrieval system and new approach to display results of query, which enables the user to learn insect distribution in a visualized way. Besides, the introduction of WAP renders convenience for those users who work in open country. The user is only required to have a WAP phone to log on the system and could be able to consult needed information. The system provides a function "create a field trip route" which help analyze and decide a field trip route.

\section{ACKNOWLEDGEMENTS}

We wish to express our thanks to Prof. Yuan Feng and Yuan Xiangqun, Northwest A \& F University, for their valuable suggestions to accomplish this system. The authors thank the reviewers for their kindly comments and suggestions. This research was supported by the special fund of Northwest A \& F University (project code: 080807; 08080209). 


\section{REFERENCES}

Lu Huimeng, Huang Yuan 2003. Design and Complementation of Expert System for Classification Retrieval and Diagnosis for China ACRIDOIDEA (ESCA). Journal of ACTA ZOOTAXONOMICA SINICA, 28: 428-433 (in Chinese).

Sun Guanying, Chen Xinxue, Cheng Jiaan 2002. Lucid: A Multi-way Expert System for Identification and Diagnosis. Journal of ACTA ZOOTAXONOMICA SINICA, 27: 871875 (in Chinese).

Xu Xiaoguo, Mo Jianchu, Cheng Jiaan 2004. Design and Development of Web System for Isoptera Taxonomy, Journal of ENTOMOTAXONOMIA, 26: 86-90 (in Chinese). 